\title{
Clinical Features of Eosinophilic Esophagitis
}

\author{
Stephan Miehlke \\ Center for Digestive Diseases, Internal Medicine Center Eppendorf, Hamburg, Germany
}

\author{
Key Words \\ Eosinophilic esophagitis · Dysphagia · Bolus obstruction . \\ Endoscopy
}

\begin{abstract}
Eosinophilic esophagitis (EoE) may affect individuals at any age with a predominance for Caucasian males. The clinical manifestation of EoE is strongly age dependent. While dysphagia and food impaction are typical lead symptoms in adults and adolescents, infants often present with unspecific symptoms such as feeding problems, abdominal pain and vomiting. Some EoE patients may also experience heartburn. Therefore, EoE should always be considered in cases of heartburn refractory to antireflux therapy. Concomitant allergic diseases such as asthma, rhinitis and eczema are prevalent. Peripheral eosinophilia and elevated total serum IgE values are found in up to 50 and $70 \%$ of cases, respectively. Endoscopic features of EoE are variable and none of them is pathognomonic. Frequent findings are mucosal edema, furrows, exudates and corrugated rings. These endoscopic abnormalities have high specificities (90-95\%), but low sensitivities (15-48\%). A novel grading and classification system for the endoscopic assessment of EoE has been proposed which includes fixed rings, exudates, furrows and edema as major features. This classification system demonstrated good interobserver agreement among pediatric and adult
\end{abstract}

\section{KARGER}

E-Mail karger@karger.com

www.karger.com/ddi gastroenterologists, and presents a useful tool to standardize endoscopic assessments and to further investigate the relation between endoscopic manifestation, clinical activity and response to treatment in EoE. Long-term follow-up studies have shown that EoE is a chronic disease causing recurrent dysphagia in the majority of cases. The prevalence of strictures significantly increases with the duration of disease, which stresses the importance of early diagnosis and consequent treatment of EoE.

(c) 2014 S. Karger AG, Basel

\section{Introduction}

Eosinophilic esophagitis (EoE) is an increasingly recognized, chronic and antigen/immune-driven inflammatory disease of the esophagus [1]. Since the first description in the literature some 20 years ago, the number of studies on EoE has increased exponentially. Meanwhile, EoE has evolved into the second most common cause of chronic esophagitis after gastroesophageal reflux disease (GERD), and now represents the primary cause of dysphagia in young male patients. Finally, it is considered to be the most common of all eosinophilic gastrointestinal disorders.

EoE is predominantly found in westernized countries and geographical areas with higher socioeconomic devel-
(C) 2014 S. Karger AG, Basel

0257-2753/14/0322-0061\$39.50/0 
Table 1. Similarities and differences in the clinical appearance of pediatric and adult EoE (adapted from [5])

\begin{tabular}{lll}
\hline & Adults & Children \\
\hline Symptoms & $\begin{array}{l}\text { dysphagia (solid food), food } \\
\text { impaction, retrosternal pain }\end{array}$ & $\begin{array}{l}\text { abdominal pain, chest pain, heartburn, } \\
\text { coughing, decreased appetite, food } \\
\text { refusal, anorexia, dysphagia, nausea, } \\
\text { regurgitation, sleeping difficulties }\end{array}$ \\
\hline
\end{tabular}

\begin{tabular}{llc}
\hline Demographics & \multicolumn{2}{c}{ male Caucasians predominant } \\
\hline Physical exam & normal & sometimes failure to thrive \\
\hline Allergic predisposition & airborne allergens predominant & food allergens predominant \\
\hline Concomitant allergic diseases & & asthma, eczema, allergic rhinitis \\
\hline
\end{tabular}

opment. However, the disease has been reported to affect individuals of every race, gender and age [2-4]. The current knowledge of the etiology and the underlying mechanisms of eosinophilic esophagitis is still limited. Although there appear to be distinct differences between eosinophilic esophagitis in pediatric patients and adults, it is generally accepted as the same disease entity $[5,6]$

\section{Symptom Presentation of EoE}

EoE can affect individuals of any age and has in fact been described in patients ranging in age from 1 to 98 years [3]. Throughout the literature, EoE has been described as a predominantly male disorder both in children and adults, being at last three times more prevalent in males than in females [2-4]. The clinical presentation of EoE is strongly dependent on the patient's age and ability to communicate symptoms. In fact, more than any other aspect of the disease, the symptoms leading to endoscopy and diagnosis vary considerably between children and adults (table 1). In children suffering from EoE, the symptom pattern is rather unspecific, including dyspepsia, heartburn or abdominal pain as the most common symptoms [3]. However, pediatric patients may additionally complain about nausea, regurgitation, chest pain or sialorrhea. Finally, decreased appetite, food aversion, failure to thrive, sleep disturbances and respiratory complaints may be associated with pediatric EoE.

In contrast, adolescent and adult EoE patients usually present with dysphagia for solid foods and/or bolus impaction. In a study of 400 consecutive adult patients presenting for routine endoscopy, EoE was found in $6.5 \%$ of the cases [7]. In this cohort, EoE was significantly associated with dysphagia, food impaction, male gender, age $<50$ years and asthma. Several series have reported dysphagia as the leading symptom in more than $70 \%$ of cases. However, food impaction has been the symptom that most often led to the diagnosis $[3,8-10]$. Food impaction is often associated with acute severe retrosternal and chest pain that can potentially lead to immediate hospitalization and emergency endoscopy, which in turn is associated with a higher risk of complications. With increasing awareness for EoE in the future, it is likely that dysphagia itself will become the most prominent symptom leading to a rapid diagnosis of EoE in adults. Dysphagia may also be a quite early event in the development of EoE as suggested by a case of a 44-year-old woman who rapidly developed a full clinical picture of de novo EoE including severe dysphagia, confluent white exudates on endoscopy and severe eosinophilic inflammation of esophageal biopsies within the first 4 weeks of sublingual immunotherapy for hay fever [11].

In clinical practice it is important to realize that EoE patients often adapt long-term coping strategies to guarantee feeding and to avoid bothering symptoms. Adult EoE patients usually do not show any clinical signs of malnutrition at diagnosis or during the evolution of the disease. In the evaluation of patients with suspected EoE, it is therefore recommended to use more sophisticated questions in tools to identify the presence and the burden of dysphagia. Questions such as 'Do you wash food down with liquids?', 'Do you chew your food for a long time?', 'Do you avoid foods such as meat or breads?' or 'Are you usually the last one to leave the table?' may be helpful in this matter [5]. 
Fig. 1. Endoscopic manifestations in adult EoE patients enrolled in a European multicenter trial: white exudate (a), longitudinal furrows (b), diffuse edema (c), fixed rings (d), severe stricture (e), and rings, furrows and edema (f) [14].
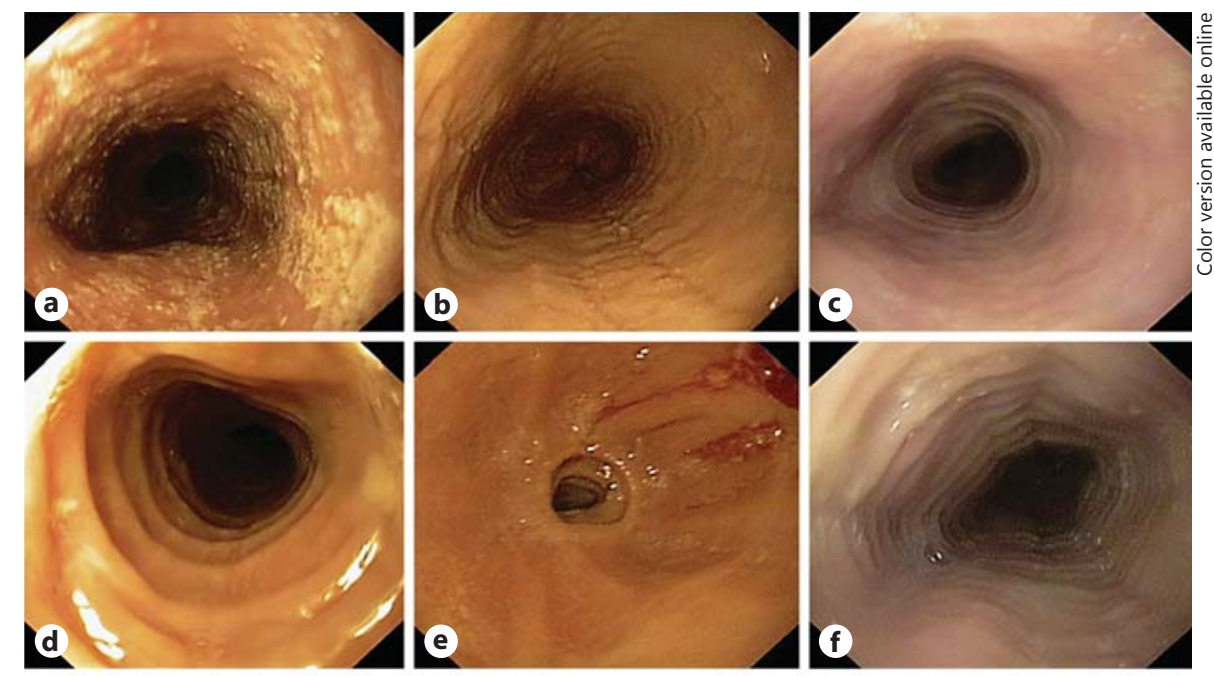

Reflux symptoms are quite common in adult EoE patients; however, the prevalence rates differ among studies and range from 9 to $94 \%[4,12,13]$. In a recent European multicenter study of 76 adult EoE patients [14], one third of the patients reported reflux symptoms and to a similar magnitude retrosternal pain or odynophagia in addition to the more prevalent and prominent dysphagia (fig. 1; table 2).

\section{Natural Course of Disease}

Little is known about the natural course and the longterm outcome of EoE. In analogy to other inflammatory bowel diseases, it can be assumed that EoE may be a progressive disease which, if untreated, may lead to irreversible long-term structural damage of the esophagus. However, the knowledge about progression of stricture formation in EoE is limited. In an early prospective study, 30 adult EoE patients were followed for a mean of 7 years without active medical therapy [15]. In this cohort, both dysphagia and esophageal eosinophilia persisted in the vast majority of patients while the overall severity of dysphagia and eosinophilia declined only marginally. A more recent retrospective study in 220 patients of the Swiss EoE database shed more light on the natural history of EoE by demonstrating a significant association between the diagnostic delay and the prevalence of esophageal strictures [16]. For example, the prevalence of esophageal strictures was $17 \%$ in patients who were diagnosed within 2 years of symptom onset and increased up to $71 \%$
Table 2. Clinical presentation of adult EoE patients enrolled in a European multicenter trial [14]

\begin{tabular}{lc}
\hline Patients & 76 \\
Males, \% & 83 \\
Mean age (range), years & $39.7(18-70)$ \\
Mean BMI (range) & $24.8(19.0-36.7)$ \\
Symptoms, \% & \\
$\quad$ Dysphagia & 100.0 \\
Food impaction & 65.8 \\
Odynophagia & 35.5 \\
Retrosternal pain & 38.3 \\
Heartburn & 32.9 \\
Regurgitation & 35.5 \\
Abdominal pain & 18.4 \\
Diarrhea & 7.8 \\
Weight loss & 2.6 \\
Allergic comorbidities, \% & 64.5 \\
Allergic rhinitis & 50.0 \\
Allergic conjunctivitis & 19.7 \\
Allergic asthma & 19.8 \\
Atopic eczema & 9.2 \\
Food allergies & 21.1 \\
Duration of dysphagia $>1$ year, \% & 50 \\
Established disease, \% & 64 \\
Previous steroids, \% & \\
Fluticasone & 22.4 \\
Budesonide & 3.9 \\
Prednisolone & 6.6 \\
Previous dilatation, \% & 13 \\
\hline
\end{tabular}


Fig. 2. Natural course of EoE: retrospective study in 200 adult EoE patients [16].

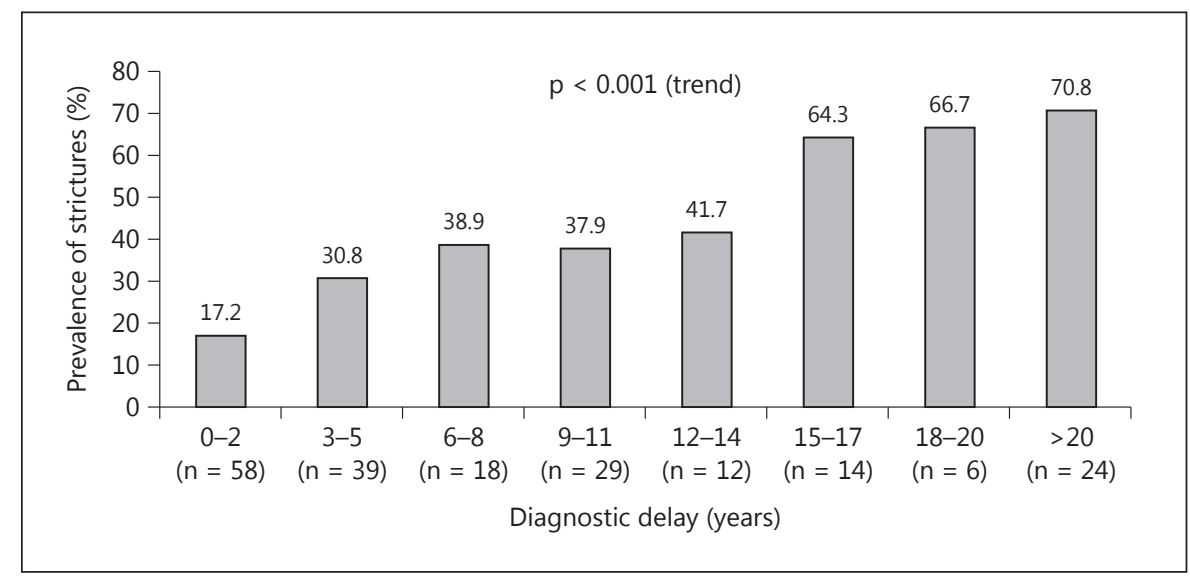

in patients with a diagnostic delay of more than 20 years (fig. 2). Diagnostic delay turned out to be the only risk factor for strictures at the time of EoE diagnosis. These data clearly indicate that it is of utmost importance to minimize the diagnostic delay in EoE in order to avoid potentially irreversible structural damage of the esophagus. So far, it seems that EoE itself is not associated with a decreased life expectancy or with an increased risk of developing malignant or premalignant lesions; however, more observational long-term studies are clearly required to confirm this.

\section{Differential Diagnosis}

Due to a potential symptom overlap, GERD represents the clinically most common and most relevant differential diagnosis to EoE $[9,17]$. Gastroesophageal reflux may by itself cause esophageal eosinophilia, and patients with GERD may also suffer from dysphagia in addition to their reflux symptoms. On the other hand, EoE may lead to an impaired function of the lower esophageal sphincter, which in turn may promote increased gastroesophageal reflux $[18,19]$. Finally, a subgroup of EoE patients does substantially respond to proton pump inhibitor (PPI) therapy, both clinically and histologically [20]. This observation had led to the term 'PPI-responsive esophageal eosinophilia' [1]. Therefore, the differentiation between EoE and GERD often remains a considerable challenge in clinical practice. This issue has been addressed in a retrospective study of 23 adult EoE and 20 adult GERD patients in which patient age, gender, weight loss, atopy, dysphagia, bolus impaction, PPI refractory heartburn, odynophagia, peripheral eosinophilia and serum IgE were subjected to logistic regression analysis [21]. As a result, the optimal differentiation between EoE and GERD was possible by the equation blood eosinophilia + bolus impaction + PPIrefractory heartburn for EoE. If at least 2 of these factors were present, the sensitivity and specificity for the diagnosis of EoE was 91 and $100 \%$, respectively.

Other differential diagnoses include eosinophilic gastroenteritis, hypereosinophilic syndromes, infectious or drug-induced esophagitis, autoimmune diseases and Crohn's disease [1].

\section{Allergy Testing in EoE}

EoE is clearly associated with allergies in both children and adults [22]. Comparable to other published series, allergic comorbidities including allergic rhinitis, conjunctivitis, asthma and atopic eczema were found in $65 \%$ of adult patients participating in a European multicenter trial [14]. Peripheral eosinophilia and elevated serum IgE levels are usually found in 50 and $75 \%$ of patients, respectively [1]. Food-specific IgE or skin prick test results may be positive in over $80 \%$ of adult EoE patients [23]; however, elimination of foods that gave positive results failed to achieve disease remission, and response to a food elimination diet has been observed in patients who had negative allergy test results. Furthermore, in adult EoE patients who responded to a six-food elimination diet and relapsed after step-wise food reintroduction, a prick test was predictive for the responsible food allergen in only a minority of cases $[13,24]$. These findings suggest dissociation between IgE-based food allergy test results and true EoE trigger foods. Therefore, allergy testing can be used to help identify food sensitizations associated with 
Fig. 3. Diagnostic validity of endoscopic features of EoE - meta-analysis of 100 studies including 4,678 EoE patients [29]. $\mathrm{PPV}=$ Positive predictive value; $\mathrm{NPV}=$ negative predictive value.

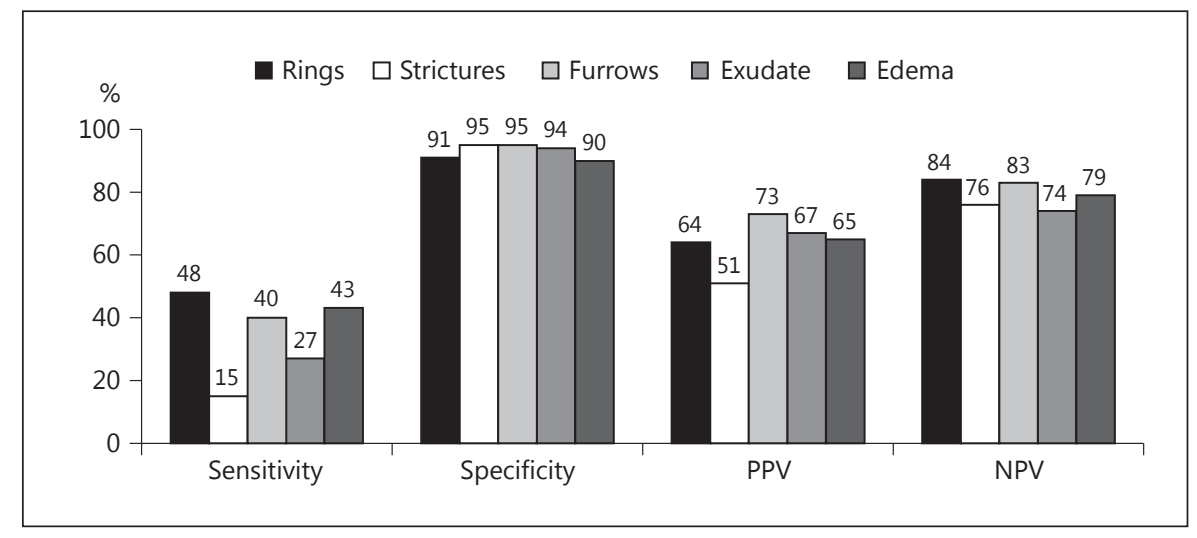

EoE, but none of the currently available techniques has proven useful or reliable for the management of EoE in clinical practice.

\section{Impact of EoE and Patient Quality of Life}

Data on health-related quality of life (QoL) in EoE patients are still sparse. QoL assessment in pediatric EoE is particularly challenging because certain aspects often expressed by diseased children themselves may not be captured by standard questionnaires or because the patient's concerns may differ from the concerns reported by their parents. QoL was assessed in a case-control study of EoE patients in early adulthood more than 15 years after the histological diagnosis of EoE [25]. Three out of 4 patients had persistent dysphagia and food impaction as adults and expressed a significantly worse QoL than matched chronic esophagitis and healthy control subjects. Furthermore, EoE has a great impact on several psychologi$\mathrm{cal}$ and social domain functions. Current research aims to develop and validate an adult eosinophilic esophagitis QoL questionnaire as a reliable disease-specific measure of health-related QoL based on 37 items with 5 subscales [26]. A recent study with 64 EoE patients showed that $69 \%$ of the patients experienced psychosocial problems including social difficulties (64\%), anxiety (41\%), sleep difficulties (33\%), depression (28\%) and school problems (26\%). Adjustment problems were identified in $44 \%$ of the patients and were significantly more common in older than in younger patients [27]. Anxious behavior and depressive feelings increased with age. These findings indicate that psychosocial evaluation should be included in the management of EoE patients, especially in pediatrics and adolescents.
Table 3. Endoscopic classification and grading of EoE (modified from [30])

$\begin{array}{ll}\text { Agreement } & \kappa \text { value } \\ \text { experts/ } & \text { experts/ } \\ \text { nonexperts, \% } & \text { nonexperts }\end{array}$

Major features

Fixed rings

Mild/moderate/severe

$69 / 71$

$0.50 / 0.50$

Also referred as concentric rings,

corrugated esophagus, corrugated

rings, ringed esophagus, trachealization

\begin{tabular}{lll}
\hline Exudates & & \\
Mild/severe & $81 / 74$ & $0.61 / 0.45$ \\
Also referred to as white spots, plaques & & \\
\hline
\end{tabular}

Furrows

Mild/severe

$79 / 81$

$0.48 / 0.57$

Also referred to as vertical lines,

longitudinal furrows

\section{Edema}

Mild/severe

$90 / 77$

$0.64 / 0.33$

Also referred to as mucosal pallor,

decreased vascular markings

\section{Minor features}

Crepe-paper esophagus

Stricture

$98 / 90$

$0.85 / 0.50$

Feline esophagus

$82 / 78$

$0.56 / 0.49$

Narrow-caliber esophagus

$66 / 67$

$0.10 / 0.12$

$80 / 73$

$0.38 / 0.29$

\section{Endoscopic Manifestations of EoE}

Upper endoscopy is the first and most important step in the diagnostic work-up of patients with dysphagia. A considerable number of endoscopic findings have been described in EoE; however, none of them can be regarded as specific or pathognomonic $[1,17,28]$. These features 
can appear in random combination and represent evidence for active inflammation and/or fibrosis. A subset of EoE patients may even present with an endoscopically normal-looking esophagus. Therefore, clinicians need to maintain a high degree of suspicion and to always take multiple esophageal biopsies, even if the mucosa appears to be normal [1]. Endoscopic features such as mucosal edema, white exudates or longitudinal furrows are generally regarded as signs of active inflammation, while strictures, corrugated rings and stenoses indicate the presence of fibrosis and tissue remodeling. There are no systematic studies to compare endoscopic features of EoE between pediatric and adult patients. However, the general impression is that signs of active inflammation are more common in children while signs of fibrosis and remodeling are more frequently observed in adult patients, suggesting a progressive course of disease in a considerable number of patients $[5,6]$.

The validity of the endoscopic features of edema, exudates, furrows, strictures and rings were investigated in a meta-analysis including 100 studies with 4,678 EoE patients and 2,742 controls (fig. 3) [29]. While these signs showed an insufficient sensitivity (15-48\%), the specificity was impressively high (90-95\%). As a result, the positive and negative predictive values ranged from 51 to $73 \%$ and from 74 to $84 \%$, respectively. The meta-analysis fur- ther showed that at least one endoscopic sign was found in $83 \%$ of the patients, while endoscopy was considered normal in $17 \%$ of patients. Interestingly, the percentage of normal endoscopies was significantly lower in prospective compared to retrospective studies (7 vs. $20 \%$ ), suggesting an increasing awareness of the endoscopist to look for subtle changes in the esophageal mucosa.

With the aim to further improve and standardize the endoscopic diagnosis of EoE, a novel endoscopic classification and grading system has been proposed as a result of a validation study of endoscopic videos from $25 \mathrm{EoE}$ patients, which were reviewed by 21 adult and pediatric gastroenterologists with various experience levels [30]. The proposed system defines a common nomenclature of and severity scores for the assessment of EoE activity and has shown good interobserver agreement for the major endoscopic EoE features of rings, exudate, furrows and edema (table 3 ). This classification and grading system will certainly be useful for future clinical trials and will facilitate standardization and comparison of endoscopic findings in clinical practice.

\section{Disclosure Statement}

The author received honoraria from Dr. Falk Pharma.

\section{References}

1 Liacouras CA, Furuta GT, Hirano I, Atkins D, Attwood SE, Bonis PA, Burks AW, Chehade M, Collins MH, Dellon ES, Dohil R, Falk GW, Gonsalves N, Gupta SK, Katzka DA, Lucendo AJ, Markowitz JE, Noel RJ, Odze RD, Putnam PE, Richter JE, Romero Y, Ruchelli E, Sampson HA, Schoepfer A, Shaheen NJ, Sicherer SH, Spechler S, Spergel JM, Straumann A, Wershil BK, Rothenberg ME, Aceves SS: Eosinophilic esophagitis: updated consensus recommendations for children and adults. J Allergy Clin Immunol 2011;128:3-20.

-2 Prasad GA, Alexander JA, Schleck CD, Zinsmeister AR, Smyrk TC, Elias RM, Locke GR 3rd, Talley NJ: Epidemiology of eosinophilic esophagitis over three decades in Olmsted County, Minnesota. Clin Gastroenterol Hepatol 2009;7:1055-1061.

3 Kapel RC, Miller JK, Torres C, Aksoy S, Lash R, Katzka DA: Eosinophilic esophagitis: a prevalent disease in the United States that affects all age groups. Gastroenterology 2008; 134:1316-1321.

4 Hruz P, Straumann A, Bussmann C, Heer P, Simon HU, Zwahlen M, Beglinger C, Schoep- fer AM, Swiss EoE Study Group: Escalating incidence of eosinophilic esophagitis: a 20year prospective, population-based study in Olten County, Switzerland. J Allergy Clin Immunol 2011;128:1349-1350.

5 Straumann A, Aceves SS, Blanchard C, Collins MH, Furuta GT, Hirano I, Schoepfer AM, Simon D, Simon HU: Pediatric and adult eosinophilic esophagitis: similarities and differences. Allergy 2012;67:477-490.

6 Lucendo AJ, Sánchez-Cazalilla M: Adult versus pediatric eosinophilic esophagitis: important differences and similarities for the clinician to understand. Expert Rev Clin Immunol 2012:8:733-745.

7 Veerappan GR, Perry JL, Duncan TJ, Baker TP, Maydonovitch C, Lake JM, Wong RK, Osgard EM: Prevalence of eosinophilic esophagitis in an adult population undergoing upper endoscopy: a prospective study. Clin Gastroenterol Hepatol 2009;7:420-426.

8 Prasad GA, Talley NJ, Romero Y, Arora AS, Kryzer LA, Smyrk TC, Alexander JA: Prevalence and predictive factors of eosinophilic esophagitis in patients presenting with dys- phagia: a prospective study. Am J Gastroenterol 2007;102:2627-2632.

$\checkmark 9$ Katzka DA: Demographic data and symptoms of eosinphilic esophagitis in adults. Gastrointest Endosc Clin N Am 2008;18:25-32.

10 Sperry SL, Crockett SD, Miller CB, Shaheen NJ, Dellon ES: Esophageal foreign-body impactions: epidemiology, time trends, and the impact of the increasing prevalence of eosinophilic esophagitis. Gastrointest Endosc 2011; 74:985-991.

11 Miehlke S, Alpan O, Schröder S, Straumann A: Induction of eosinophilic esophagitis by sublingual pollen immunotherapy. Case Rep Gastroenterol 2013;7:363-368.

12 Liacouras CA, Spergel JM, Ruchelli, et al: Eosinophilic esophagitis: a 10-year experience in 381 children. Clin Gastroenterol Hepatol 2005;3:1198-1206.

13 Gonsalves N, Yang GY, Doerfler B, Ritz S, Ditto AM, Hirano I: Elimination diet effectively treats eosinophilic esophagitis in adults; food reintroduction identifies causative factors. Gastroenterology 2012;142: 1451-1459. 
14 Miehlke S, Hruz P, von Arnim U, Madisch A, Vieth M, Bussmann C, Bajbouj M, Fibbe C, Wittenburg H, Allescher HD, Reinshagen M, Schubert S, Tack J, Mueller R, Dilger K, Greinwald R, Straumann A: Two new budesonide formulations are highly efficient for treatment of active eosinophilic esophagitis: results from a randomized, double-blind, double-dummy, placebo-controlled multicenter trial. DDW 2014, accepted.

15 Straumann A, Spichtin HP, Grize L, Bucher KA, Beglinger C, Simon HU: Natural history of primary eosinophilic esophagitis: a followup of 30 adult patients for up to 11.5 years Gastroenterology 2003;125:1660-1669.

-16 Schoepfer AM, Safroneeva E, Bussmann C, Kuchen T, Portmann S, Simon HU, Straumann A: Delay in diagnosis of eosinophilic esophagitis increases risk for stricture formation, in a time-dependent manner. Gastroenterology 2013;145:1230-1236.e2.

17 Müller S, Pühl S, Vieth M, Stolte M: Analysis of symptoms and endoscopic findings in 117 patients with histological diagnosis of eosinophilic esophagitis. Endoscopy 2007;39:339344.

18 Kwiatek MA, Hirano I, Kahrilas PJ, Rothe J, Luger D, Pandolfino J: Mechanical properties of the esophagus in eosinophilic esophagitis. Gastroenterology 2011;140:82-90.
9 Roman S, Hirano I, Kwiatek MA, Gonsalves N, Chen J, Kahrilas PJ, Pandolfino JE: Manometric features of eosinophilic esophagitis in esophageal pressure topography. Neurogastroenterol Motil 2011;23:208-214.

20 Molina-Infante J, Ferrando-Lamana L, Ripoll C, Hernandez-Alonso M, Mateos JM, Fernandez-Bermejo $M$, Dueñas $C$, FernandezGonzalez N, Quintana EM, Gonzalez-Nuñez MA: Esophageal eosinophilic infiltration responds to proton pump inhibition in most adults. Clin Gastroenterol Hepatol 2011;9: 110-117.

21 von Arnim U, Wex T, Röhl FW, Neumann H, Küster D, Weigt J, Mönkemüller K, Malfertheiner P: Identification of clinical and laboratory markers for predicting eosinophilic in adults. Digestion 2011;84:323-327.

22 Roy-Ghanta, Larosa DF, Katzka DA: Atopic characteristics of adult patients with eosinophilic esophagitis. Clin Gastroenterol Hepatol 2008;6:531-535.

23 Simon D, Marti H, Heer P, Simon HU, Braathen LR, Straumann A: Eosinophilic esophagitis is frequently associated with IgE-mediated allergic airway diseases. J Allergy Clin Immunol 2005;115:1090-1092.

24 Lucendo AJ, Arias A, Gonzalez-Cervera J, Yague-Compadre JL, Guagnozzi D, Angueira T, Jimnez-Contreras S, Gonzalez-Castillo S, Rodriguez-Domingez B, De Rezende LC, Tenias JM: Empiric 6-food elimination diet induced and maintained prolonged remission in patients with adult eosinophilic esophagitis: a prospective study on the food cause of the disease. J Allergy Clin Immunol 2013;131: 797-804.
25 DeBrosse CW, Franciosi JP, King EC, et al: Long-term outcomes in pediatric onset esophageal eosinophilia. J Allergy Clin Immunol 2011;128:132-138.

26 Taft TH, Kern E, Kwiatek MA, Hirano I, Gonsalves N, Keefer L: The adult eosinophilic oesophagitis quality of life questionnaire: a new measure of health-related quality of life. Aliment Pharmacol Ther 2011;34:790-798.

27 Harris RF, Menard-Katcher C, Atkins D, Furuta GT, Klinnert MD: Psychosocial dysfunction in children and adolescents with eosinophilic esophagitis. J Pediatr Gastroenterol Nutr 2013;57:500-505.

28 Straumann A, Spichtin HP, Bucher KA, Heer P, Simon HU: Eosinophilic esophagitis: red on microscopy, white on endoscopy. Digestion 2004;70:109-116.

29 Kim HP, Vance RB, Shaheen NJ, Dellon ES: The prevalence and diagnostic utility of endoscopic features of eosinophilic esophagitis: a meta-analysis. Clin Gastroenterol Hepatol 2012;10:988-996.

30 Hirano I, Moy N, Heckman MG, Thomas CS, Gonsalves N, Achem SR: Endoscopic assessment of the oesophageal features of eosinophilic oesophagitis: validation of a novel classification and grading system. Gut 2013;62: 489-495. 